\title{
An energy-equilibrium model for complex stress effect on fatigue crack initiation
}

\author{
ZHAO SiCong, XIE JiJia ${ }^{*}$, ZHAO AiGuo \& WU XiaoLei \\ State Key Laboratory of Nonlinear Mechanics, Institute of Mechanics, Chinese Academy of Sciences, Beijing 100190, China
}

Received December 3, 2012; accepted February 18, 2013; published online March 14, 2014

\begin{abstract}
Based on Tanaka and Mura's fatigue model and Griffith theory for fracture, an energy-equilibrium model was proposed to explain the complex stress effect on fatigue behavior. When the summation of the elastic strain energy release and the stored strain energy of accumulated dislocations reach the surface energy of a crack, the fatigue crack will initiate in materials. According to this model, for multiaxial stress condition, the orientation of the crack initiation and the initiation life can be deduced from the energy equilibrium equation. For the uniaxial fatigue loading with mean stress, the relation between the maximum stress or the minimum stress and the stress amplitude is in agreement with an ellipse equation on the constant life diagram. If the ratio of the mean stress to stress amplitude is less than a critical value -0.17 , and the stress amplitude keeps constant, the fatigue crack initiation life will decrease with the increase of the compress mean stress. In this model, the mean stress does not cause damage accumulation with the fatigue cycles in crack initiation. For this reason, the loading sequence of different load levels would induce the cumulative damage to deviate from the Palmgren-Miner cumulative damage rule. The procedure of estimating the damage under random loading is also discussed.
\end{abstract}

fatigue initiation, mean stress effect, multiaxial fatigue, Tanaka-Mura model, damage accumulation

PACS number(s): 46.50.+a, 62.20.Mk, 81.40.Np

Citation: Zhao S C, Xie J J, Zhao A G, et al. An energy-equilibrium model for complex stress effect on fatigue crack initiation. Sci China-Phys Mech Astron, 2014, 57: 916-926, doi: 10.1007/s11433-013-5241-z

\section{Nomenclature}

a Half grain size

$\alpha \quad$ Tilt angle of the normal direction of a plane from the tension direction

$\Delta U \quad$ Accumulated dislocation energy per half cycle

$W_{\mathrm{s}} \quad$ Specific fracture energy

$K_{\mathrm{I}}, K_{\mathrm{II}}$ Stress intensity factor of mode I and mode II cracks

$G \quad$ Elastic strain energy release rate for creating unit area of crack

$S \quad$ Area of crack surface

$\sigma_{n} \quad$ Normal stress

*Corresponding author (email: xiejj@1nm.imech.ac.cn) $\sigma_{\mathrm{m}} \quad$ Mean tensile stress

$\sigma_{\mathrm{a}} \quad$ Amplitude of tensile stress

$\sigma_{\mathrm{e}} \quad$ Endurance limit

$\sigma_{\text {uts }} \quad$ Ultimate tensile strength

$\mu \quad$ Shear modulus

A $2 \mu W_{\mathrm{s}} /\left[(1-v) \pi k^{2} a\right]$

$(\alpha, \beta)$ Orientation angles of an arbitrary plane

$p \quad$ Factor for slip irreversibility

$k \quad$ Resistance of dislocation movement

$\tau_{\mathrm{m}} \quad$ Mean shear stress

$\tau_{\mathrm{a}} \quad$ Amplitude of shear stress

$\tau \quad$ Shear stress

$\Delta \tau \quad$ Shear stress range

$T \quad$ Loading period

$N_{\mathrm{f}} \quad$ Fatigue cycles for crack initiation 


\section{Poisson's ratio}

Phase difference between the tensile stress and the shear stress Parameter for combination of complex loading.

\section{Introduction}

Fatigue failure is one of the most common modes of failure for mechanical components. In general cases, components in service are subject to complex stress, such as a loading with mean stress or multiaxial stress. However, most of the fatigue data are measured under uniaxial loading condition. So, it is very important to develop the criterion of fatigue failure for samples under complex loading. Then the stress-life curve of uniaxial loading could be used to assess the fatigue life of components under complex loading.

The investigations of mean stress effect of fatigue behavior are traced to the early time of the fatigue research [1-6]. The relationships of Goodman, Morrow, and Gerber can be found in any textbook on fatigue. However, all these methods are empirical methods. The micro mechanism of fatigue damage with the mean stress is not reflected in these models.

Similar to the investigations of mean stress effect, many different methods [7-9] were proposed on the assessment of the fatigue life under multiaxial fatigue. You and Lee [8] classified these theories into five types, i.e. empirical formulas and modifications of the Coffin-Manson equation; application of stress or strain invariants; use of the space averages of stress or strain; critical plane approaches; and use of energy which has accumulated on the materials under consideration. Of these different methods, the method of critical plane approaches [9] is the most popular for multiaxial fatigue assessment because of its effectiveness and broad applications. Sines, Findley, McDiarmid, Dang Van, etc. [7], proposed the respective stress-based critical plane models for fatigue damage criteria. In brief, a linear or nonlinear combination of shear stresses and normal stresses acting on the critical plane was calculated to be an equivalent of uniaxial loading. The orientation of the critical plane was also determined with different critical plane concepts, such as the plane of maximum shear stress or the plane of maximum shear stress range. Similar to those based on stress analysis, the approaches based on strain analysis were also proposed by Brown and Miller, Fatemi and Socie, Smith, Watson and Topper [7], respectively. All these methods succeeded in some cases of multiaxial fatigue assessment. However, these models were also empirical. Parameters in these models should be determined by sufficient experimental data for different materials. The mechanism of fatigue damage for multiaxial fatigue is not very clear in these models.

In general, for polycrystalline metals or alloys, the micro mechanism of fatigue damage is related to the extrusion or intrusion along the persisting slide band in the grains $[2,10,11]$. Fatigue crack would initiate at these sites. Based on dislocation accumulation with cyclic loading, Mura and coworkers proposed a model [12-14] for fatigue crack initiation. Chan [15] extended Tanaka and Mura's model to include crack size and relevant micro structural parameters in the equations. However, in these models, the parameter of stress is only the shear stress range on the slip plane. So, these models can not be used to describe the fatigue under the loading case with mean stress or multiaxial stress.

Although the fatigue life varies with the loading condition, the micro mechanism of fatigue damage must be the same. So, there must be a unified equation to describe the fatigue life under different loading conditions, including the mean stress effect and multiaxial loading. Based on this point and Tanaka \& Mura's fatigue model, this paper attempts to propose a modified Energy-Equilibrium method for assessment of fatigue with complex stress. This new model provides a unified criterion for multiaxial fatigue. The mean stress effect and multiaxial stress effect are discussed in detail.

\section{Model and analysis}

\subsection{Energy-equilibrium model}

In 1981, Tanaka and Mura [12] proposed a dislocation model for fatigue crack initiation. They pointed out that if the stored strain energy due to dislocations accumulated after $n$-cycles became equal to the surface energy, a crack with the length of grain size $2 a$ would initiate.

$$
2 N_{\mathrm{f}} \Delta U=4 a W_{\mathrm{s}}
$$

where $N_{\mathrm{f}}$ is the cycle of loading for crack initiation, $\Delta U$ is the accumulated energy per half cycle, $a$ is the half length of the grain size, and $W_{\mathrm{s}}$ is the specific fracture energy for a unit area.

Later, Mura and coworkers $[13,14]$ developed their model based on free energy considerations. The total free energy of the system increases with fatigue cycle number due to the increase in stored elastic strain energy of accumulated dislocations. When the Gibbs free energy change attains a maximum at a critical number of cycles, a micro crack would initiate. In this modified model, the mechanical energy release was added in the energy equilibrium equation. However, in their paper, the mechanical energy release is related to the stress range, but not the maximum stress.

As described in Griffith's fracture theory, there is always elastic strain energy release with the crack initiation in solid. So the elastic strain energy release with the forming of a fatigue crack is not negligible. Based on this point, we work out a modified energy balance equation for fatigue crack initiation as follows: 


$$
\int_{0}^{S} G_{\max } \mathrm{d} S+2 N_{\mathrm{f}} \Delta U=2 S W_{\mathrm{s}}
$$

where, $G$ is the elastic strain energy release rate for creating unit area of crack. The first item at the left of the equation is the maximum value of virtual elastic strain energy release in one cycle of loading for creating a crack with an area of $S$. Obviously, this value should be determined by the maximum loading instead of the loading range in one cycle. The second item at the left side of the equation is the dislocation energy accumulated with the number of the cycles, which is the same as the item in Tanaka-Mura's model. The item at the right side of the equation is the surface energy for creating a fatigue crack with an area of $S$. That is to say, if the sum of the elastic strain energy release and stored strain energy due to dislocations accumulated after n-cycles becomes equal to the surface energy, a fatigue crack will initiate.

For complex conditions, stress tensor varies with the time. This means that not only the value but also the direction of the main stress changes with the time. And the stress condition on an arbitrary plane also varies with the time.

As shown in Figure 1(a), for an arbitrary plane $(\alpha, \beta)$, the normal stress $\sigma_{n}$ and the shear stress $\tau$ will be solved by using coordination transform. According to the value of the normal stress $\sigma_{n}$ and the shear stress $\tau$, the maximum of virtual elastic strain energy release in one cycle of loading for creating a crack of area $S$ in this plane could be calculated by using linear elastic fracture mechanics method. Here, if $\sigma_{n}$ is compressed, there will be no mode I type elastic strain energy release.

The accumulated dislocation energy per half cycle can be estimated with the maximum range of shear stress in this plane. As shown in Figure 1(b), in the plane $(\alpha, \beta)$ the shear stress $\tau$ changes with the time. In one cycle, the trace of the shear force vector forms a closed curve in the plane. The maximum caliper diameter is the range of the shear stress in this plane.

Then, according to eq. (2), the fatigue crack initiation life $N_{\mathrm{f}}$ in terms of the loading parameters $\sigma_{n}, \tau$ and $\Delta \tau$ on the plane $(\alpha, \beta)$ could be produced. The minimum of the fatigue life $N_{\mathrm{f}}$ with the change of the orientation $(\alpha, \beta)$ is the fatigue crack initiation life of the sample. And the plane $(\alpha$, $\beta$ ) which is related to the minimum of the fatigue life $N_{\mathrm{f}}$ is the orientation of the fatigue crack.

\subsection{Tension-Torsion fatigue crack initiation}

For 3-D crack initiation, the analysis is very complicated. However, for Tension-Torsion fatigue, it's relatively simple. From eq. (2), we have

$$
\begin{aligned}
& \frac{(1-v)}{2 \mu} \int_{0}^{a}\left(K_{I}^{2}+K_{I I}^{2}\right)_{\max } \mathrm{d}(2 a)+2 N_{\mathrm{f}} \Delta U=4 a W_{\mathrm{s}} \\
& \Rightarrow \frac{(1-v) C^{2} \pi\left(\sigma_{n}^{2}+\tau^{2}\right)_{\max } a^{2}}{2 \mu} \\
& +2 N_{\mathrm{f}} \frac{(1-v) \pi(\Delta \tau-2 k)^{2} a^{2}}{2 \mu}=4 a W_{\mathrm{s}} \\
& \Rightarrow C^{2}\left(\sigma_{n}^{2}+\tau^{2}\right)_{\max }+2 N_{\mathrm{f}}(\Delta \tau-2 k)^{2}=\frac{8 \mu W_{\mathrm{s}}}{(1-v) \pi a},
\end{aligned}
$$

where $\sigma_{n}$ is the normal stress on crack surface, $\tau$ is the shear stress on crack surface, $k$ is the resistance of dislocation movement on the slide plane, $\Delta \tau$ is the range of shear stress on crack surface in one cycle of loading, and it must be greater than the $2 k$. $C$ is a geometrical factor of the crack. Here, if $\sigma_{n}$ is compressed, then there will be no elastic strain energy release of Mode I.

Consider a sample subject to the tension and torsion force, as shown in Figure 2. The waveform of loading is
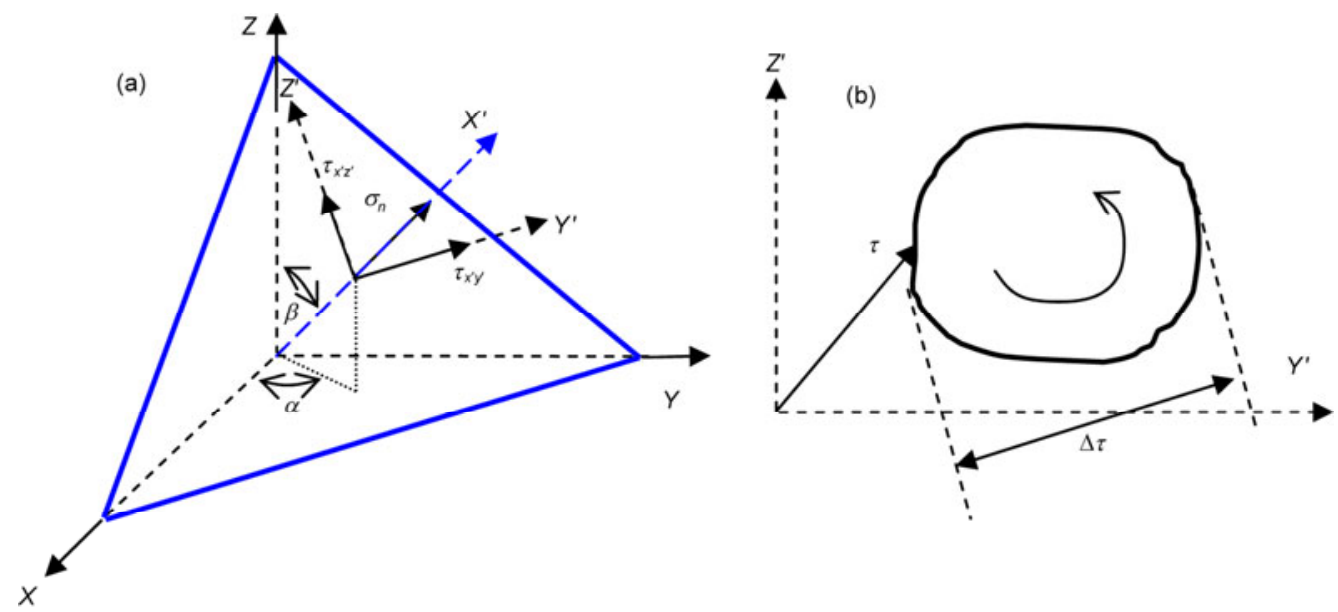

Figure 1 (Color online) (a) Stresses acting on a plane in the sample signed as plane $(\alpha, \beta)$, where $\alpha$, $\beta$ were orientation angles of the normal direction of this plane in a three-dimensional coordinate system on the sample; (b) the trace of shear stress vector on the plane ( $\alpha, \beta)$ in a cycle forms a closed curve. The range of the shear stress is the maximum caliper diameter of the area surrounded by the curve. 


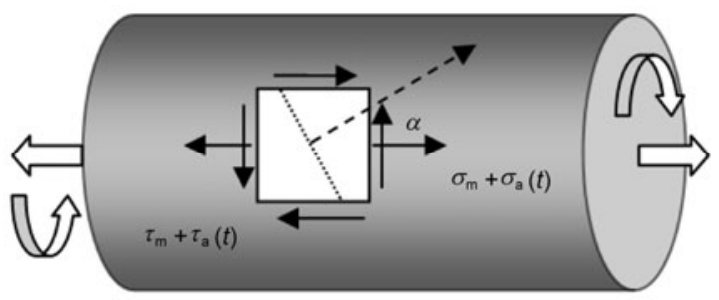

Figure 2 A schematic illustration of stresses acting on the surface of a rod under tension-torsion fatigue loading.

sinusoidal and the periods of tension stress and shear stress are the same. The stress tensor is as follows:

$$
\begin{aligned}
\sigma_{i j} & =\left[\begin{array}{ll}
\sigma_{11} & \sigma_{12} \\
\sigma_{21} & \sigma_{22}
\end{array}\right] \\
& =\left[\begin{array}{cc}
\sigma_{\mathrm{m}}+\sigma_{\mathrm{a}} \sin \left(\frac{2 \pi t}{T}\right) & \tau_{\mathrm{m}}+\tau_{\mathrm{a}} \sin \left(\frac{2 \pi t}{T}+\theta\right) \\
\tau_{\mathrm{m}}+\tau_{\mathrm{a}} \sin \left(\frac{2 \pi t}{T}+\theta\right) & 0
\end{array}\right],
\end{aligned}
$$

where $\sigma_{\mathrm{m}}, \sigma_{\mathrm{a}}$ are the mean stress and the amplitude of tension stress respectively, and $\tau_{\mathrm{m}}, \tau_{\mathrm{a}}$ are the mean stress and the amplitude of shear stress respectively. $\theta$ is the phase difference between the tension stress and the shear stress, and $T$ is the period of loading.

For a plane, in which the normal direction tilts an angle $\alpha$ from the tension direction, the normal stress and the shear stress are calculated as follows:

$$
\begin{aligned}
\sigma_{n}= & \left(\sigma_{\mathrm{m}}+\sigma_{\mathrm{a}} \sin \left(\frac{2 \pi t}{T}\right)\right) \cos ^{2}(\alpha) \\
& +\left(\tau_{\mathrm{m}}+\tau_{\mathrm{a}} \sin \left(\frac{2 \pi t}{T}+\theta\right)\right) \sin (2 \alpha), \\
\tau= & -\left(\sigma_{\mathrm{m}}+\sigma_{\mathrm{a}} \sin \left(\frac{2 \pi t}{T}\right)\right) \cos \alpha \sin \alpha \\
& +\left(\tau_{\mathrm{m}}+\tau_{\mathrm{a}} \sin \left(\frac{2 \pi t}{T}+\theta\right)\right) \cos 2 \alpha
\end{aligned}
$$

The range of shear stress on plane $\alpha$ is

$$
\begin{aligned}
& \Delta \tau \\
& =2 \sqrt{\left(-\sigma_{a} \cos \alpha \sin \alpha+\tau_{a} \cos 2 \alpha \cos \theta\right)^{2}+\left(\tau_{a} \cos 2 \alpha \sin \theta\right)^{2}} .
\end{aligned}
$$

Obviously, the normal stress and the shear stress in this plane vary with the time. The virtual elastic strain energy release of modes I and II also changes with the time in one loading cycle.

If the normal stress is tensile, the elastic strain energy release includes mode I and mode II part. Otherwise, there is only mode II part. As mentioned in eq. (2), the virtual elas- tic strain energy release should be the maximum of the possible values in one loading cycle.

Then, put the maximum value of the elastic strain energy release and the range of shear stress in this plane into the eq. (3), and the possible fatigue life of this plane can be deduced.

Finally, the fatigue life of the sample is the minimum value of all the fatigue life with different orientations.

In eq. (3), both items at the left side is related to orientation angle $\alpha$ and the first item-elastic strain energy release should be the maximum of the possible values in one loading cycle. Hence, the analytical solution is very complicated, except for samples under uniaxial fatigue loading. Numerical simulation is more practical for samples under common tension-torsion fatigue loading.

\subsection{Tensile fatigue loading with mean stress}

For the sample under uniaxial tensile fatigue loading, the stress tensor takes the form:

$$
\sigma_{i j}=\left[\begin{array}{ll}
\sigma_{11} & \sigma_{12} \\
\sigma_{21} & \sigma_{22}
\end{array}\right]=\left[\begin{array}{cc}
\sigma_{\mathrm{m}}+\sigma_{\mathrm{a}} \sin \left(\frac{2 \pi t}{T}\right) & 0 \\
0 & 0
\end{array}\right] .
$$

From eqs. (5)-(7), the normal stress and the shear stress on the plane $\alpha$ are calculated as follows:

$$
\begin{gathered}
\sigma_{n}=\left(\sigma_{\mathrm{m}}+\sigma_{\mathrm{a}} \sin \left(\frac{2 \pi t}{T}\right)\right) \cos ^{2}(\alpha), \\
\tau=-\left(\sigma_{\mathrm{m}}+\sigma_{\mathrm{a}} \sin \left(\frac{2 \pi t}{T}\right)\right) \cos \alpha \sin \alpha .
\end{gathered}
$$

And the range of the shear stress on the plane $\alpha$ is

$$
\Delta \tau=\sigma_{\mathrm{a}} \sin 2 \alpha
$$

If there is $\sigma_{\mathrm{m}} \geqslant 0$, the maximum value of elastic energy release is at the $T / 4$, when the normal stress is tensile in an arbitrary plane $\alpha$. So, in this case, there is joint mode I and mode II elastic strain energy release.

From eq. (3), we have

$$
\begin{aligned}
& \frac{(1-v) \pi a^{2} C^{2}\left(\sigma_{\mathrm{m}}+\sigma_{\mathrm{a}}\right)^{2} \cos ^{2}(\alpha)}{2 \mu} \\
& +2 N_{\mathrm{f}} \frac{(1-v) \pi a^{2}\left(\sigma_{\mathrm{a}} \sin (2 \alpha)-2 k\right)^{2}}{2 \mu} \\
& =4 a W_{\mathrm{s}} \\
& \Rightarrow C^{2}\left(\sigma_{\mathrm{m}}+\sigma_{\mathrm{a}}\right)^{2} \cos ^{2}(\alpha)+2 N_{\mathrm{f}}\left(\sigma_{\mathrm{a}} \sin (2 \alpha)-2 k\right)^{2} \\
& =\frac{8 \mu W_{\mathrm{s}}}{(1-v) \pi a}
\end{aligned}
$$




$$
\begin{aligned}
& \Rightarrow \frac{\left(\left(\bar{\sigma}_{\mathrm{m}}+\bar{\sigma}_{\mathrm{a}}\right) \cos (\alpha)\right)^{2}}{\left(\frac{\sqrt{A}}{C}\right)^{2}}+\frac{\left(\bar{\sigma}_{\mathrm{a}} \sin (2 \alpha)-1\right)^{2}}{\left(\sqrt{\frac{A}{2 N_{\mathrm{f}}}}\right)^{2}}=1 \\
& \Rightarrow \frac{\left(\bar{\sigma}_{\max } \cos (\alpha)\right)^{2}}{\left(\frac{\sqrt{A}}{C}\right)^{2}}+\frac{\left(\bar{\sigma}_{\mathrm{a}} \sin (2 \alpha)-1\right)^{2}}{\left(\sqrt{\frac{A}{2 N_{\mathrm{f}}}}\right)^{2}}=1,
\end{aligned}
$$

where stress is normalized by the twice frictional stress for dislocation movement.

$$
\bar{\sigma}_{\mathrm{m}}=\frac{\sigma_{\mathrm{m}}}{2 k}, \quad \bar{\sigma}_{\mathrm{a}}=\frac{\sigma_{\mathrm{a}}}{2 k}, \quad A=\frac{2 \mu W_{s}}{(1-v) \pi k^{2} a} .
$$

This equation demonstrates that, in any plane $\alpha$, the maximum stress and the amplitude stress are in agreement with an ellipse equation.

The fatigue life $N_{\mathrm{f}}$ is determined from eq. (12) as:

$$
N_{\mathrm{f}}=\frac{A-C^{2} \bar{\sigma}_{\max }^{2} \cos ^{2}(\alpha)}{2\left(\bar{\sigma}_{\mathrm{a}} \sin (2 \alpha)-1\right)^{2}} \text {. }
$$

Obviously, the fatigue crack initiation life changes with the direction of the plane. Let the derivative of $N_{\mathrm{f}}$ equals zero, and we have

$$
\begin{aligned}
N_{\mathrm{f}}{ }^{\prime}= & 0 \Rightarrow 2 A \bar{\sigma}_{\mathrm{a}} \operatorname{tg}^{4} \alpha-C^{2} \bar{\sigma}_{\max }^{2} \operatorname{tg}^{3} \alpha \\
& -C^{2} \bar{\sigma}_{\max }^{2} \operatorname{tg} \alpha+2 C^{2} \bar{\sigma}_{\max }^{2} \bar{\sigma}_{\mathrm{a}}-2 A \bar{\sigma}_{\mathrm{a}}=0 .
\end{aligned}
$$

Root of eq. (14) gives out the direction angle $\alpha$ of the crack initiation surface. According to the results of numerical simulation with different parameters, this tilt angle $\alpha$ is less than but close to the 45 degree, which is related to the plane with maximum shear stress.

If $\sigma_{\mathrm{m}} \leqslant-\sigma_{\mathrm{a}}$, the normal stress in an arbitrary plane $\alpha$ is always compressed in the loading cycle. So, in this case, there is only mode II elastic energy release and the maximum value of virtual elastic strain energy release appears at the $3 T / 4$.

From eq. (3), we have

$$
\begin{aligned}
& \frac{(1-v) \pi a^{2} C^{2}\left(\sigma_{\mathrm{m}}-\sigma_{\mathrm{a}}\right)^{2} \cos ^{2}(\alpha) \sin ^{2}(\alpha)}{2 \mu} \\
& \quad+2 N_{\mathrm{f}} \frac{(1-v) \pi a^{2}\left(\sigma_{\mathrm{a}} \sin (2 \alpha)-2 k\right)^{2}}{2 \mu} \\
& =4 a W_{\mathrm{s}} \\
& \Rightarrow C^{2}\left(\sigma_{\mathrm{m}}-\sigma_{\mathrm{a}}\right)^{2} \cos ^{2}(\alpha) \sin ^{2}(\alpha) \\
& \quad+2 N_{\mathrm{f}}\left(\sigma_{\mathrm{a}} \sin (2 \alpha)-2 k\right)^{2} \\
& =\frac{8 \mu W_{\mathrm{s}}}{(1-v) \pi a}
\end{aligned}
$$

$$
\begin{aligned}
& \Rightarrow \frac{\left(\left(\bar{\sigma}_{\mathrm{m}}-\bar{\sigma}_{\mathrm{a}}\right) \cos (\alpha) \sin (\alpha)\right)^{2}}{\left(\frac{\sqrt{A}}{C}\right)^{2}}+\frac{\left(\bar{\sigma}_{\mathrm{a}} \sin (2 \alpha)-1\right)^{2}}{\left(\sqrt{\frac{A}{2 N_{\mathrm{f}}}}\right)^{2}}=1 \\
& \Rightarrow \frac{\left(\bar{\sigma}_{\min } \sin (2 \alpha)\right)^{2}}{\left(\frac{2 \sqrt{A}}{C}\right)^{2}}+\frac{\left(\bar{\sigma}_{\mathrm{a}} \sin (2 \alpha)-1\right)^{2}}{\left(\sqrt{\frac{A}{2 N_{\mathrm{f}}}}\right)^{2}}=1 .
\end{aligned}
$$

In this case, the tilt angle $\alpha$ of fatigue crack initiation plane is always the 45 degree.

So, we have

$$
\frac{\left(\bar{\sigma}_{\min }\right)^{2}}{\left(\frac{2 \sqrt{A}}{C}\right)^{2}}+\frac{\left(\bar{\sigma}_{\mathrm{a}}-1\right)^{2}}{\left(\sqrt{\frac{A}{2 N_{\mathrm{f}}}}\right)^{2}}=1 .
$$

If $-\sigma_{\mathrm{a}} \leqslant \sigma_{\mathrm{m}} \leqslant 0$, the maximum value of virtual elastic strain energy release is at the $T / 4$ or $3 T / 4$. In one cycle of loading, when $t=T / 4$, the normal stress is tensile, so the elastic strain energy release includes mode I and mode II. When $t=3 T / 4$, the normal stress is compressed, and the elastic energy strain release includes only mode II. So, if

$$
\frac{G_{T / 4}}{G_{3 T / 4}}=\frac{\left(K_{I}^{2}+K_{I I}^{2}\right)_{T / 4}}{\left(K_{I I}^{2}\right)_{3 T / 4}}=\frac{\left(\sigma_{\mathrm{m}}+\sigma_{\mathrm{a}}\right)^{2}}{\left(\sigma_{\mathrm{m}}-\sigma_{\mathrm{a}}\right)^{2} \sin ^{2}(\alpha)} \geqslant 1 .
$$

Then, the maximum value of virtual elastic energy release appears at the $T / 4$.

That is to say, if

$$
\begin{aligned}
& \frac{\left(\sigma_{\mathrm{m}}+\sigma_{\mathrm{a}}\right)^{2}}{\left(\sigma_{\mathrm{m}}-\sigma_{\mathrm{a}}\right)^{2}} \geqslant \sin ^{2}(\alpha) \\
& \Rightarrow 0 \leqslant \alpha \leqslant \arcsin \left(\frac{\left(\sigma_{\mathrm{m}}+\sigma_{\mathrm{a}}\right)}{\left(\sigma_{\mathrm{a}}-\sigma_{\mathrm{m}}\right)}\right),
\end{aligned}
$$

the fatigue life $N_{\mathrm{f}}(\alpha)$ is solved by using eq. (13), otherwise using eq. (16). As shown in Figure 3, eq. (18) gives the boundary line. At the left side of the boundary line, eq. (16) is used to calculate the fatigue initiation life. Because the fatigue initiation plane is always at or close to the 45 degree, a critical ratio of the mean stress to the stress amplitude can be calculated as follows.

For $\alpha=\pi / 4$, from eq. (18), we have

$$
\frac{\sigma_{\mathrm{m}}}{\sigma_{\mathrm{a}}}=\frac{1-\sqrt{2}}{1+\sqrt{2}} \approx-0.17 \text {. }
$$

If the ratio of the mean stress to the stress amplitude is less than the critical value, eq. (16) will be used to solve the fatigue life. Otherwise eq. (12) will be used.

Furthermore, according to Tanaka and Mura's model 


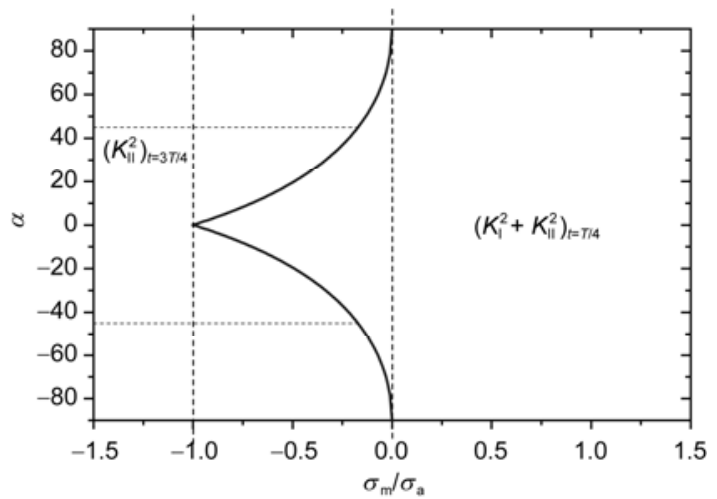

Figure 3 The boundary between the area of using eq. (12) and the area of using eq. (16) for the calculation of the elastic strain energy release.

[12], the range of shear stress must be greater than twice the frictional stress of dislocations on the slide plane. So, for the tilt angle $\alpha$, we must have

$$
\begin{aligned}
& \bar{\sigma}_{\mathrm{a}} \sin (2 \alpha)-1>0 \\
& \Rightarrow \frac{1}{2} \arcsin \left(\frac{1}{\bar{\sigma}_{\mathrm{a}}}\right)<\alpha<\frac{\pi}{2}-\frac{1}{2} \arcsin \left(\frac{1}{\bar{\sigma}_{\mathrm{a}}}\right) .
\end{aligned}
$$

That means, for fatigue under uniaxial loading, the fatigue initiation plane will not appear at the 0 degree or 90 degree tilt from the loading direction.

\subsection{Torsion fatigue loading with mean stress}

For the sample under torsion fatigue loading, the stress tensor takes the form

$$
\begin{aligned}
\sigma_{i j} & =\left[\begin{array}{ll}
\sigma_{11} & \sigma_{12} \\
\sigma_{21} & \sigma_{22}
\end{array}\right] \\
& =\left[\begin{array}{cc}
0 & \tau_{\mathrm{m}}+\tau_{\mathrm{a}} \sin \left(\frac{2 \pi t}{T}\right) \\
\tau_{\mathrm{m}}+\tau_{\mathrm{a}} \sin \left(\frac{2 \pi t}{T}\right) & 0
\end{array}\right] .
\end{aligned}
$$

From eqs. (5)-(7), the normal stress and the shear stress on the plane $\alpha$ are calculated as follows:

$$
\begin{gathered}
\sigma_{n}=\left(\tau_{m}+\tau_{a} \sin \left(\frac{2 \pi t}{T}\right)\right) \sin (2 \alpha), \\
\tau=\left(\tau_{\mathrm{m}}+\tau_{\mathrm{a}} \sin \left(\frac{2 \pi t}{T}\right)\right) \cos (2 \alpha) .
\end{gathered}
$$

And the range of the shear stress on the plane $\alpha$ is

$$
\Delta \tau=2 \tau_{\mathrm{a}}|\cos (2 \alpha)|
$$

From eq. (3), we have

$$
\begin{aligned}
& \frac{(1-v) \pi a^{2} C^{2}\left(\tau_{\mathrm{m}} \pm \tau_{\mathrm{a}}\right)^{2}}{2 \mu} \\
& \quad+2 N_{\mathrm{f}} \frac{(1-v) \pi a^{2}\left(2 \tau_{\mathrm{a}}-2 k\right)^{2}}{2 \mu}=4 a W_{\mathrm{s}} \\
& \Rightarrow C^{2}\left(\tau_{\mathrm{m}} \pm \tau_{\mathrm{a}}\right)^{2}+2 N_{\mathrm{f}}\left(2 \tau_{\mathrm{a}}-2 k\right)^{2}=\frac{8 \mu W_{\mathrm{s}}}{(1-v) \pi a} \\
& \Rightarrow \frac{\left(\bar{\tau}_{\mathrm{m}} \pm \bar{\tau}_{\mathrm{a}}\right)^{2}}{\left(\frac{\sqrt{A}}{C}\right)^{2}}+\frac{\left(2 \bar{\tau}_{\mathrm{a}}-1\right)^{2}}{\left(\sqrt{\frac{A}{2 N_{\mathrm{f}}}}\right)^{2}}=1,
\end{aligned}
$$

where if $\bar{\tau}_{\mathrm{m}}>0$, there is $\tau_{\mathrm{m}}+\tau_{\mathrm{a}}$ used at the left side of this equation. Otherwise, there is $\tau_{\mathrm{m}}-\tau_{\mathrm{a}}$. In this condition, the fatigue crack will always initiate along the 0 degree or 90 degree, which is the plane of maximum shear stress.

\section{Discussion}

\subsection{The parameters in the model}

In Tanaka and Mura's model [12], there are some parameters of materials, such as: specific fracture energy $W_{\mathrm{s}}$, grain size $2 a$ and frictional stress of the dislocation $k$. It's difficult to determine all these parameters directly in practice. However, if the sample is only loaded under a monotonic increasing tensile stress untill break, the maximum of possible tensile stress is the ultimate tensile strength of the sample, and the orientation of fracture surface would be 45 degree tilt to the loading axial for ductile metals. Then, from eq. (12), we have

$$
A=\frac{\bar{\sigma}_{\mathrm{uts}}^{2}}{2}
$$

where $C=1$. And the ultimate tensile strength $\bar{\sigma}_{\text {uts }}$ is normalized by twice the frictional stress for dislocation movement. For ductile metals, in addition to the ultimate tensile strength $\sigma_{\text {uts }}$, the true fracture strength $\tilde{\sigma}_{f}$ or the fatigue strength coefficient $\sigma_{f}^{\prime}$ maybe other possible choice in eq. (26).

The frictional stress of dislocation $k$ gives a fatigue limit. That means that if the shear stress is less than the frictional stress, there will be no fatigue damage. So, endurance limit $\sigma_{\mathrm{e}}$ can be used to substitute it.

In Tanaka and Mura's model [12], it is assumed that the dislocations formed by each cycle of loading are irreversible. This assumption is not rational, especially for the case when the shear stress range is very close to twice the frictional stress of dislocation. This assumption leads to the calculated fatigue life being less than the experiment results for several orders. Mura and Nakasone [14] discussed the reversibility 
of dislocations and proposed a parameter $f$ in their extension model where, $f$ varies from 0 to 1 , representing complete reversibility to complete irreversibility of slip. Mughrabi $[10,11]$ proposed a similar parameter $p$ for the cyclic slip irreversibility. $p$ will be zero when the slip is entirely reversible, and it will be equal to one when the slip is entirely irreversible.

According to all these considerations, eq. (12) will take the following form

$$
\left(C \sigma_{\max } \cos (\alpha)\right)^{2}+2 p N_{\mathrm{f}}\left(\sigma_{\mathrm{a}} \sin (2 \alpha)-\sigma_{\mathrm{e}}\right)^{2}=\sigma_{\mathrm{uts}}^{2} / 2 .
$$

In eq. (27), the parameter $p$ is a constant factor for slip irreversibility. However, as mentioned by Mughrabi [11], the slip irreversibility was found to vary significantly in magnitude from case to case. Higher loading amplitudes would induce a higher irreversibility. Obviously, the loading frequency and the temperature are also important for slip irreversibility. The farther reseach of the effects of all these parameters are out of this paper, and should be investigated in the future work.

Assume that the relation between the slip irreversibility and the loading amplitudes is a power law function:

$$
p=B\left(\frac{\sigma_{\mathrm{a}}}{\sigma_{\mathrm{e}}}-1\right)^{m} \text {. }
$$

Here, $B$ and $m$ are constants for slip irreversibility. Then eq. (27) will take the form as follows:

$$
\begin{aligned}
& \frac{\sigma_{\max }^{2}}{2 \sigma_{\mathrm{e}}^{2}}+2 B N_{\mathrm{f}}\left(\frac{\sigma_{\mathrm{a}}}{\sigma_{\mathrm{e}}}-1\right)^{2+m}=\frac{\sigma_{\mathrm{uts}}^{2}}{2 \sigma_{\mathrm{e}}^{2}} \\
& \Rightarrow \sigma_{\mathrm{a}}=\sigma_{\mathrm{e}}+\sigma_{\mathrm{e}}\left(\frac{\sigma_{\text {uts }}^{2}-\sigma_{\max }^{2}}{2 B \sigma_{\mathrm{e}}^{2}}\right)^{\frac{1}{2+m}}\left(2 N_{\mathrm{f}}\right)^{-\frac{1}{2+m}} .
\end{aligned}
$$

Here, $C=1$ and the crack initiates alone 45 degree tilt plane which is the plane with the maximum shear stress. This formula is similar to Basquin's equation. Here, the fatigue strength coefficient and the Basquin exponent take the form as follows:

$$
\sigma_{\mathrm{f}}^{\prime}=\sigma_{\mathrm{e}}\left(\frac{\sigma_{\mathrm{uts}}^{2}-\sigma_{\max }^{2}}{2 B \sigma_{\mathrm{e}}^{2}}\right)^{\frac{1}{2+m}}, \quad b=-\frac{1}{2+m} .
$$

\subsection{Mean stress effect}

For the case of $\frac{\sigma_{\mathrm{m}}}{\sigma_{a}}>\frac{1-\sqrt{2}}{1+\sqrt{2}}$, with the increase of the normal tensile stress, the tilt angle $\alpha$ would decrease from 45 degree to a smaller degree. However, in the long life fatigue regime, this angle is very close to 45 degree. So, from eq. (12), the relation between the maximum stress and the amplitude stress agrees with the ellipse curve.

$$
\frac{\bar{\sigma}_{\max }^{2}}{2\left(\frac{\sqrt{A}}{C}\right)^{2}}+\frac{\left(\bar{\sigma}_{\mathrm{a}}-1\right)^{2}}{\left(\sqrt{\frac{A}{2 N_{\mathrm{f}}}}\right)^{2}}=1
$$

In addition, from eq. (27), the relation between the mean stress and the amplitude stress takes the form as follows:

$$
\begin{aligned}
& \frac{\left(\sigma_{\mathrm{m}}+\sigma_{\mathrm{a}}\right)^{2}}{\left(\frac{\sigma_{\mathrm{UTS}}}{C}\right)^{2}}+\frac{\left(\sigma_{\mathrm{a}}-\sigma_{\mathrm{e}}\right)^{2}}{\left(\frac{\sigma_{\mathrm{UTS}}}{2 \sqrt{P N_{\mathrm{f}}}}\right)^{2}}=1 \quad \text { if } \frac{\sigma_{\mathrm{m}}}{\sigma_{\mathrm{a}}} \geqslant \frac{1-\sqrt{2}}{1+\sqrt{2}}, \\
& \frac{\left(\sigma_{\mathrm{m}}-\sigma_{\mathrm{a}}\right)^{2}}{\left(\frac{\sqrt{2} \sigma_{\mathrm{UTS}}}{C}\right)^{2}}+\frac{\left(\sigma_{\mathrm{a}}-\sigma_{\mathrm{e}}\right)^{2}}{\left(\frac{\sigma_{\mathrm{UTS}}}{2 \sqrt{P N_{\mathrm{f}}}}\right)^{2}}=1 \quad \text { if } \quad \frac{\sigma_{\mathrm{m}}}{\sigma_{\mathrm{a}}}<\frac{1-\sqrt{2}}{1+\sqrt{2}} .
\end{aligned}
$$

The constant life diagram of this model is shown in Figure 4. Compared to other models, this model gives an extended constant life diagram including compress mean stress. In this diagram, if the stress amplitude keeps constant, the fatigue initiation life will increase with the decrease of the mean stress when $\frac{\sigma_{\mathrm{m}}}{\sigma_{\mathrm{a}}}>\frac{1-\sqrt{2}}{1+\sqrt{2}}$, and will decrease with the decrease of the mean stress when $\frac{\sigma_{\mathrm{m}}}{\sigma_{\mathrm{a}}} \leqslant \frac{1-\sqrt{2}}{1+\sqrt{2}}$. That is to say, the compress mean stress is not always beneficial to preventing fatigue crack initiation, although it is beneficial to decreasing the fatigue crack propagation rate.

In practice, there would be friction between the crack surfaces when there is normal compress stress on this plane. Due to the friction of crack surface, the value of the elastic

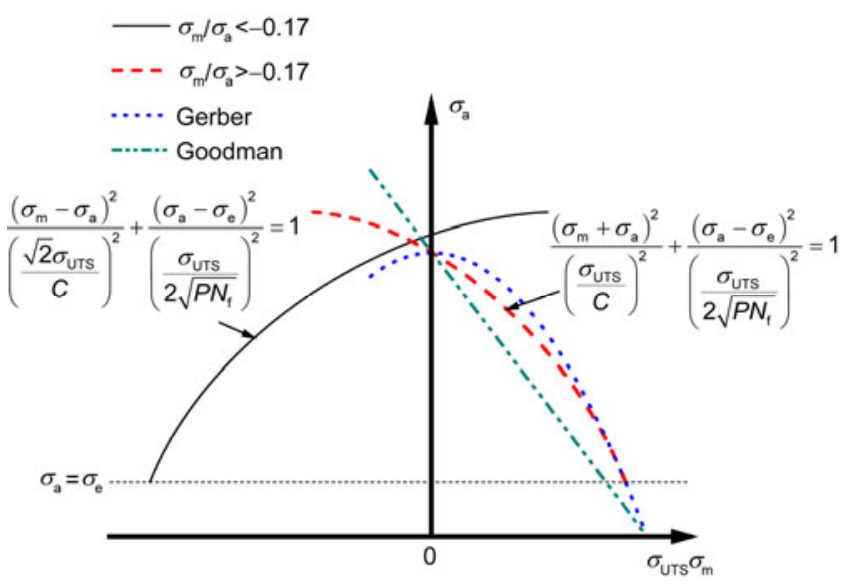

Figure 4 (Color online) A schematic illustration of constant life diagram of this model. If $\sigma_{\mathrm{m}} / \sigma_{\mathrm{a}} \geqslant-0.17$, the constant life curve would be described by eq. (32), the dash curve in this figure. Otherwise, the curve would be described by eq. (33), the solid curve in this figure. The curves described by Gerber relationship and Goodman relationship are also shown as comparison. 
energy release of Mode II may be very difficult to calculate. Although eq. (33) is very simple in physical mechanism, the analysis for fatigue crack initiation might be more complex for cases with compress stress. Eq. (33) would be the low limit for crack initiation with compress mean stress.

Dowling [3] collected the experiment data of three different alloys for the mean stress effect in his book. The comparison of these experimental data and the prediction by eq. (32) is shown in Figure 5.

From Figure 5, for all these alloys, the simulation curves fit the experiment data very well, except for Ti6Al4V when the fatigue life increases to $10^{5}$ cycles or longer. And it seems that the simulation parameter $p$ for cyclic slip irreversibility decreases with the increase of the fatigue life. The range of $p$ is from $10^{-5}$ to $10^{-8}$, which is much lower
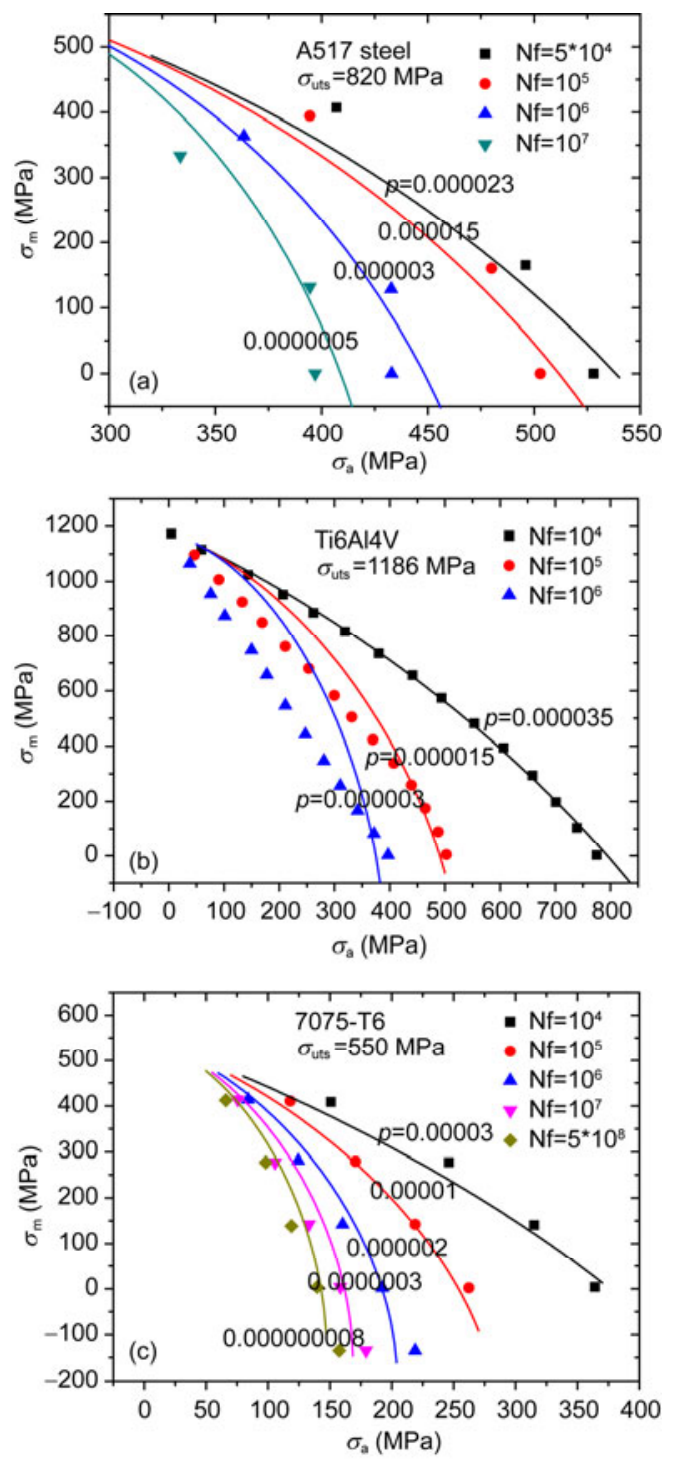

Figure 5 (Color online) Constant life diagrams for three different alloys. The symbols in these figures are experiment data from reference [3]. The continuum curves are simulation results for different fatigue life. (a) A517 steel. (b) Ti6Al4V. (c) 7075-T6. than those collected by Mughrabi [11]. In his paper, the irreversibility $p$ of some pure metals or single phase alloy including copper, $\alpha$-brass, and $\alpha$-iron varies from 1 to $10^{-5}$. Obviously, the microstructure of metallic alloys is much more complex than pure or single phase metals. The cyclic slip irreversibility of dislocations would be very sensitive to the microstructure. Because of the importance of $p$ in fatigue damage accumulation, the cyclic slip irreversibility for different metals or alloys should be investigated in the future work.

\subsection{Critical plane method for multiaxial fatigue}

Eq. (3) gives the general relationship between the fatigue initiation life and the normal stress, shear stress, and the shear stress range on an arbitrary plane. Substituting eq. (26) into eq. (3) and considering the slip irreversibility for an arbitrary plane, if the normal stress is tensile, we have

$$
\begin{aligned}
& C^{2}\left(\sigma_{n}^{2}+\tau^{2}\right)_{\max }+2 p N_{\mathrm{f}}(\Delta \tau-2 k)^{2}=\frac{\sigma_{\mathrm{uts}}^{2}}{2} \\
& \Rightarrow 4 p N_{\mathrm{f}}=\frac{\sigma_{\mathrm{uts}}^{2}-2 C^{2}\left(\sigma_{n}^{2}+\tau^{2}\right)_{\max }}{(\Delta \tau-2 k)^{2}},
\end{aligned}
$$

where the item at the right side of this equation is a nonlinear combination of the stress parameters on this plane and the material parameters. This combination is related to the virtual life for an arbitrary plane, which varies with the plane orientation.

If we define a parameter $\lambda$ as the combination of the loading condition, the complex loading could be converted to the effective uniaxial stress amplitude. Then, the fatigue life could be estimated with the $S-N$ curve under uniaxial loading.

$$
\begin{gathered}
\lambda=\frac{\sigma_{\mathrm{uts}}^{2}-2\left(\sigma_{n}^{2}+\tau^{2}\right)_{\max }}{\left(\Delta \tau-\sigma_{\mathrm{e}}\right)^{2}}=\frac{\sigma_{\mathrm{uts}}^{2}-\sigma_{\mathrm{a}}^{2}}{\left(\sigma_{\mathrm{a}}-\sigma_{\mathrm{e}}\right)^{2}} \\
\Rightarrow \sigma_{\mathrm{a}}=\frac{\lambda \sigma_{\mathrm{e}}+\sqrt{(1+\lambda) \sigma_{\mathrm{uts}}^{2}-\lambda \sigma_{\mathrm{e}}^{2}}}{1+\lambda} .
\end{gathered}
$$

Here, $\lambda$ is the parameter which is related to the fatigue life and the crack orientation. For an arbitrary plane, the orientation with the minimum value of the $\lambda$ is the tilt angle of the crack plane. For all the loading conditions, the same value of $\lambda$ means the same fatigue life.

Obviously, this model belongs to the energy model for multiaxial fatigue. And all the energies for crack initiation could be calculated with the normal stress and shear stress on an arbitrary plane. Different from other energy models for multiaxial fatigue, the virtual elastic strain energy release in this model does not cause accumulated damage with the loading cycles for crack initiation.

This model can be used for proportional or nonpropor- 
tional loading without any change in the parameters. However, it can only be used to estimate the fatigue crack initiation life for polycrystalline metals and alloys, but not the total life. Obviously, fatigue crack initiation tends to appear on the plane with maximum shear stress, while the crack propagation tends to appear alone the plane with maximum normal stress. The mechanism of damage accumulation would change in the course of crack growth. So, this model is not good enough to assess the fatigue crack growth for multiaxial fatigue.

\subsection{Damage accumulation and random fatigue}

According to Tanaka and Mura's model [12], fatigue crack will initiate when the amount of stored dislocation energy of each cycle reaches the value of surface energy. Obviously, Tanaka and Mura's model coincides with the Palmgren-Miner cumulative damage rule, which means that the load sequence has no effect on the fatigue damage accumulation. However, in this new model, the linear damage rule is not correct. Although the amount of stored dislocation energy accumulates linearly with the fatigue cycles, the virtual elastic strain energy does not produce any damage with the fatigue cycles, until the last cycle of the crack initiation.

For example, a sample is tested under two uniaxial stress conditions one after another. The first stress condition is at the maximum stress-stress amplitude combination $\left(\sigma_{\max 1}\right.$, $\left.\sigma_{\mathrm{a} 1}\right)$, and the fatigue life under this stress condition is $N_{1}$. The other stress condition is at the $\left(\sigma_{\max 2}, \sigma_{\mathrm{a} 2}\right)$, and the fatigue life under this stress condition is $N_{2}$.

According to the energy-equilibrium eq. (3), a simplified form is as follows:

$$
E_{i}+N_{i} \Delta U_{i}=E_{\mathrm{s}}
$$

where $E_{i}$ is the virtual elastic strain energy release, which is related to the maximum stress level $\sigma_{\max i} . \Delta U_{i}$ is the accumulated dislocation energy in one cycle, which is related to the stress amplitude $\sigma_{\mathrm{a} i}$. $E_{s}$ is the surface energy for producing a crack with a length of grain diameter. As shown in Figure 6 , there are two cases. In the first case, the sample is loaded under $\left(\sigma_{\max 1}, \sigma_{\mathrm{a} 1}\right)$ for $n_{1}$ cycles, then under $\left(\sigma_{\max 2}, \sigma_{\mathrm{a} 2}\right)$ for $\mathrm{n}_{2}$ cycles to break. In the second one the sample is loaded un$\operatorname{der}\left(\sigma_{\max 2}, \sigma_{\mathrm{a} 2}\right)$ for $\mathrm{n}_{2}$ cycles, then under $\left(\sigma_{\max 1}, \sigma_{\mathrm{a} 1}\right)$ for $\mathrm{n}_{1}$ cycles to break. Let $\sigma_{\max 1}<\sigma_{\max 2}$, so we have $E_{1}<E_{2}$.

From eq. (36), for the first case, we have

$$
\begin{aligned}
& E_{2}+\left(n_{2} \Delta U_{2}+n_{1} \Delta U_{1}\right)=E_{\mathrm{s}} \\
& \Rightarrow \frac{n_{1}}{N_{1}} \frac{E_{\mathrm{s}}-E_{1}}{E_{\mathrm{s}}-E_{2}}+\frac{n_{2}}{N_{2}}=1 \\
& \Rightarrow \frac{n_{1}}{N_{1}}+\frac{n_{2}}{N_{2}}<1 .
\end{aligned}
$$

For the second case, we have

$$
\begin{aligned}
& E_{1}+\left(n_{1} \Delta U_{1}+n_{2} \Delta U_{2}\right)=E_{\mathrm{s}} \\
& \Rightarrow \frac{n_{1}}{N_{1}}+\frac{n_{2}}{N_{2}} \frac{E_{\mathrm{s}}-E_{2}}{E_{\mathrm{s}}-E_{1}}=1 \\
& \Rightarrow \frac{n_{1}}{N_{1}}+\frac{n_{2}}{N_{2}}>1 .
\end{aligned}
$$

Obviously, because the possible elastic strain energy release is different for the different loading conditions, only if $\sigma_{\max 1}$ equals $\sigma_{\max 2}$, the loading sequence will induce the cumulative damage to deviate from the Palmgren-Miner cumulative damage rule.

However, this conclusion is contrary to many researchers' work [16,17] and the experiment results. A reasonable explanation is that this model is for fatigue crack initiation, but not for the crack propagation. Otherwise, in Tanaka and Mura's model, there is no load sequence effect on the damage accumulation of dislocations. Actually, as discussed earlier, the cyclic slip irreversibility would vary with the loading condition. So, the effect of loading history on evolution of dislocation should be investigated in the future more deeply.

For random fatigue, the general process includes cycle

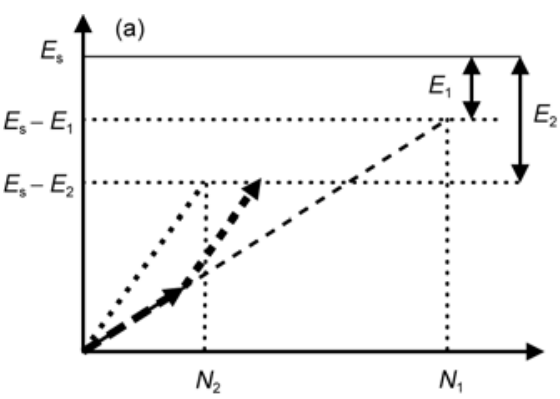

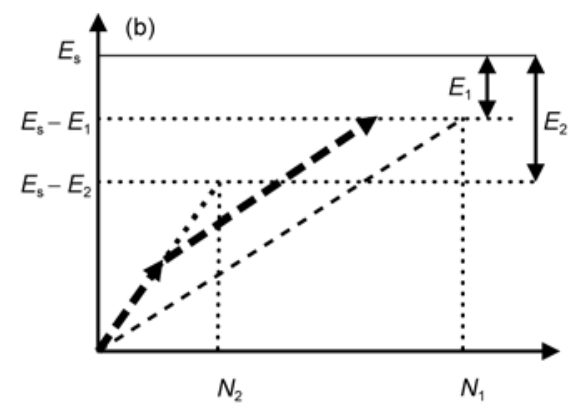

Figure 6 A schematic illustration of the energy accumulation for two different load sequences. The arrow line describes the energy accumulated with the fatigue cycles. The dash line describes that of the sample under only $\left(\sigma_{\max 1}, \sigma_{\mathrm{a} 1}\right)$, the point line describes that of the sample under only $\left(\sigma_{\max 2}, \sigma_{\mathrm{a} 2}\right)$. Here, let $\sigma_{\max 1}<\sigma_{\max 2}$, so $E_{1}<E_{2}$. (a) Sample loaded under $\left(\sigma_{\max 1}, \sigma_{\mathrm{a} 1}\right)$ for $n_{1}$ cycles, then under $\left(\sigma_{\max 2}, \sigma_{\mathrm{a} 2}\right)$ for $n_{2}$ cycles to break; (b) sample loaded under $\left(\sigma_{\max 2}, \sigma_{\mathrm{a} 2}\right)$ for $n_{2}$ cycles, then under $\left(\sigma_{\max }, \sigma_{\mathrm{a} 1}\right)$ for $n_{1}$ cycles to break. 
counting and subsequent damage accumulation calculation. Through cycle counting, a random stress history could be resolved to a set including a series of amplitude-mean stress combinations $\left(\sigma_{\mathrm{m} i}, \sigma_{\mathrm{a} i}\right)$. Then, methods proposed by Goodman, Gerber, Morrow etc. are used to convert each amplitude-mean combination to equivalent completely reversed stress amplitude $\sigma_{\text {ari }}$ respectively. Finally, from an S-N curve for stress ratio $R=-1$, the accumulated damage can be estimated by using Palmgren-Miner rule. However, as discussed earlier in this section, the mean stress does not bring accumulative damage with the fatigue cycle. According to this model, the accumulative dislocation energy is only related to the stress amplitude levels. The conversion of amplitude-mean combinations $\left(\sigma_{\mathrm{m} i}, \sigma_{\mathrm{a} i}\right)$ to $\sigma_{\text {ari }}$ would bring errors in damage calculation. However, the maximum stress $\operatorname{MAX}\left(\sigma_{\mathrm{m} i}+\sigma_{\mathrm{a} i}\right)$ or the minimum stress $\operatorname{MIN}\left(\sigma_{\mathrm{m} i}-\sigma_{\mathrm{a} i}\right)$ in the whole loading history is important, which determines the maximum possible elastic strain energy release in the fatigue crack initiation.

There are many different methods used for cycle counting. Rainflow method [2,3] might be the most popular one. From our model, the cycle counting would be much simpler. What we should do is just recording the ranges between each peak and the adjacent valley. As shown in Figure 7, the load history would be resolved to a set of simple range $\Delta \sigma_{i}$. For example, $\Delta \sigma_{1}=\left|\sigma_{\mathrm{B}}-\sigma_{\mathrm{A}}\right|$. Here, if $\Delta \sigma_{i}$ is less than twice the friction stress of dislocation, this segment should be neglected. For example, if $\Delta \sigma_{6}=\left|\sigma_{\mathrm{G}}-\sigma_{\mathrm{F}}\right|<2 k$, the segment F-G should be neglected, and there is only segment E-H rather than E-F, F-G, and G-H. The maximum stress at point $\mathrm{D}$ would be used to calculate the virtual elastic strain energy release, so it should be recorded in the cycle counting.

Based on eq. (29), the accumulative damage for random fatigue could be as follows:

$$
\frac{\sigma_{\max }^{2}}{\sigma_{\mathrm{e}}^{2}}+4 N_{f} B \sum_{i=1}^{n}\left(\frac{\Delta \sigma_{i}}{2 \sigma_{\mathrm{e}}}-1\right)^{2+m}=\frac{\sigma_{\mathrm{uts}}^{2}}{\sigma_{\mathrm{e}}^{2}} .
$$

Here, $n$ is the number of the stress range in the random

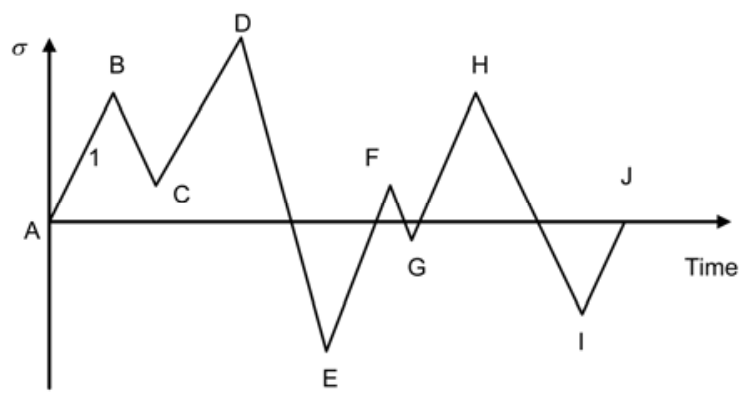

Figure 7 A schematic diagram of a random loading history with the time. The simple ranges between the peak and adjacent valley should be recorded in the cycle counting, so does the maximum stress or minimum stress in the whole wave. The simple range less than $2 k$ should be neglected. loading spectrum. $\mathrm{N}_{\mathrm{f}}$ is the repetition number of the random loading spectrum for fatigue crack initiation. $\sigma_{\max }$ is the maximum stress in the random loading spectrum.

\section{Conclusions}

Based on Tanaka and Mura's model, an energy-equilibrium model for complex stress effect on fatigue crack initiation is proposed in this paper. From this model, some conclusions reached in this investigation are as follows:

(1) The elastic strain energy and the stored dislocation energy both contribute to the fatigue crack initiation. When the summation of the accumulated stored dislocation energy and the virtual elastic strain energy release reaches the surface energy for producing a crack with the size of grain diameter, the fatigue crack will initiate.

(2) For samples under uniaxial fatigue loading, the relation between the maximum stress or the minimum stress and the stress amplitude is in agreement with an ellipse equation on an arbitrary plane.

(3) There is a critical ratio of mean stress to stress amplitude, which equals about -0.17 . If the ratio of mean stress to stress amplitude is less than the critical value, and the amplitude keeps constant, the fatigue initiation life will decrease with the decrease of the mean stress. Compress mean stress is not always beneficial to prolonging the life of crack initiation.

(4) Because of the effect of elastic strain energy release, the Palmgren-Miner rule will yield incorrect results in damage accumulation for fatigue crack initiation.

(5) The traditional procedure for estimating the damage under random loading might be incorrect, for the mean stress does not bring accumulative damage with fatigue cycles.

The authors wish to thank Prof. HONG Y S and Dr. SUN C Q of the Institute of Mechanics, Chinese Academy of Sciences for helpful discussions in this work. This work was supported by the National Basic Research Program of China (Grant No. 2012CB937500), and the National Natural Science Foundation of China (Grant Nos. 11072243 and 11202210).

1 Sendeckyj G P. Constant life diagrams - a historical review. Int J Fatigue, 2001, 23: 347-353

2 Suresh S. Fatigue of Materials. New York: Cambridge University Press, 1991. 130-132, 97-111

3 Dowling N E. Mechanical Behavior of Materials: Engineering Methods for Deformation, Fracture, and Fatigue. Englewood Cliffs, NJ: Prentice Hall, 1993. 370-376

4 Nihei M, Heuler P, Boller C, et al. Evaluation of mean stress effect on fatigue life by use of damage parameters. Int J Fatigue, 1986, 8(3): 119-126

5 Dowling $\mathrm{N}$ E, Calhoun $\mathrm{C} \mathrm{A}$, Arcari A. Mean stress effects in stress-life fatigue and the Walker equation. Fatigue Fract Eng Mater Struct, 2009, 32: 163-179

6 Dowling N E. Mean stress effects in strain-life fatigue. Fatigue Fract Eng Mater Struct, 2009, 32: 1004-1019 
7 Socie D F, Marquis G B. Multiaxial Fatigue. Warrendale, Pa.: Society of Automotive Engineers, Inc., 2000. 129-230

8 You B R, Lee S B. A critical review on multiaxial fatigue assessments of metals. Int J Fatigue, 1996, 18(4): 235-244

9 Karolczuk A, Macha E. A review of critical plane orientations in multiaxial fatigue failure criteria of metallic materials. Int J Fracture, 2005, 134: 267-304

10 Mughrabi $\mathrm{H}$. On the life-controlling microstructural fatigue mechanisms in ductile metals and alloys in the gigacycle regime. Fatigue Fract Eng Mater Struct, 1999, 22: 633-641

11 Mughrabi H. Cyclic slip irreversibilities and the evolution of fatigue damage. Metall Mater Trans A, 2009, 40A: 1257-1279

12 Tanaka K, Mura T. A dislocation model for fatigue crack initiation. J Appl Mech, 1981, 48: 97-103
13 Venkataraman G, Chung Y W, Nakasone Y, et al. Free energy formulation of fatigue crack initiation along persistent slip bands: Calculation of S-N curves and crack depths. Acta Metall Mater, 1990, 38(1): 31-40

14 Mura T, Nakasone Y. A theory of fatigue crack initiation in solids. J Appl Mech, 1990, 57: 1-6

15 Chan K S. A microstructure-based fatigue-crack-initiation model. Metall Mater Trans A, 2003, 34A: 43-58

16 Mesmacquea G, Garciab S, Amrouchea A, et al. Sequential law in multiaxial fatigue, a new damage indicator. Int J Fatigue, 2005, 27: 461-467

17 Košút J. History influence exponent in cumulative fatigue damage determined using two-step loading experiments. Fatigue Fract Eng Mater Struct, 2002, 25: 575-586 